\title{
Engagement y rendimiento académico en estudiantes de la Facultad de Ciencias Aplicadas de la Universidad Nacional del Centro del Perú
}

\section{Engagement and academic performance in students of the Faculty of Applied Sciences of Universidad Nacional del Centro del Perú}

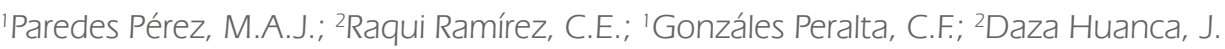 \\ Facultad de Ciencias Aplicadas, Universidad Nacional del Centro del Perú \\ Email: maparedes@uncp.edu.pe
}

\section{Resumen}

El compromiso académico es un tema de preocupación y trascendencia internacional, relacionado al desenvolvimiento de estudiantes en diferentes niveles académicos; la desmotivación y escaso involucramiento, incrementan los niveles de deserción en todos los niveles de la actividad académica. El estudio del engagement (vigor, dedicación y absorción), se ha convertido en uno de los temas principales de investigación en contextos académicos. El objetivo de la investigación, fue determinar la relación entre engagement y rendimiento académico.

Se ejecutó un censo a 298 estudiantes matriculados en el periodo lectivo 2018-I, de Administración de Negocios, Administración Hotelera y Turismo e Ingeniería Agroindustrial; el instrumento utilizado fue la escala UWES sobre engagement académico; en el caso del rendimiento se emplearon los consolidados de las tres carreras profesionales.

Los resultados indican que no existe correlación significativa entre las variables estudiadas engagement y rendimiento académico, siendo el nivel de significancia del $35 \%$ lo cual a su vez indica un margen de error alto, siendo permisible a los más un $5 \%$. La correlación de Spearman, muestra un nivel de relación de $12.3 \%$; esto indica que los estudiantes se desenvuelven con niveles de motivación interna personal; la principal conclusión, es que pese a las condiciones adversas y desmotivación mantienen un rendimiento académico equilibrado.

Palabras clave: engagement académico, rendimiento académico, estudiante universitario, burnout académico

\begin{abstract}
Academic commitment is a topic of international concern and importance related to the development of students at different academic levels; demotivation and low involvement increase the desertion levels in all the stages of the academic activity. The engagement study (vigor, dedication and absorption) has become one of the main topics of research in academic contexts; The objective of this research was determining the relationship between engagement and academic performance.

A census was carried out to 298 students enrolled in the 2018-I academic year, from Business Administration, Hotel Management and Tourism and Agroindustrial Engineering; the instrument used was the UWES scale on academic engagement; in the case of performance, the consolidated results of the three professional careers were used.

The results indicate that there is no significant correlation between the variables studied engagement and academic performance, being the level of significance of $35 \%$ which in turn indicates a high margin of error being permissible to the most $5 \%$. The Spearman correlation shows a relationship level of $12.3 \%$; This indicates that students perform with personal internal motivation levels; The main conclusion is that in spite of adverse conditions and demotivation maintain a balanced academic performance.
\end{abstract}

Keywords: academic engagement, academic performance, university student, academic burnout 


\section{Introducción}

El escaso involucramiento académico en estudiantes de muchas regiones del mundo, es un problema alarmante, puesto que se están incrementando los niveles de desmotivación y escaso involucramiento e incluso abandono de la actividad académica. El estudio del engagement (vigor, dedicación y absorción), se ha convertido en uno de los temas principales de investigación dentro de contextos académicos; sin embargo, aún existe mucho por investigar, puesto que no todos los espacios educativos son similares. El estudio del engagement y los factores que lo determinan son prácticamente escasos, al ser un tema reciente que se viene tratando a partir de la segunda década del presente siglo XXI.

Si el engagement se relaciona con el rendimiento académico, se podrán identificar las características de los factores que lo determinan de acuerdo al contexto en el cual los estudios se llevan a cabo. Una vez identificadas las características del engagement académico, se podrán desarrollar propuestas para hacer sostenible su mantenimiento dentro de los espacios educativos. Sin embargo, se determinó que no necesariamente altos niveles de engagement se relacionan con un rendimiento académico sobresaliente.

Al hablar de engagement, nos referimos a la capacidad humana de experimentar altos niveles de vigor, dedicación y absorción que un estudiante experimenta al realizar sus labores académicas; en el caso de burnout, sus características definidas son: agotamiento emocional, insatisfacción y cinismo; asimismo, rendimiento académico tiene que ver con niveles de resultados y logros expresados en calificaciones de estudiantes.

En relación al desempeño académico en estudiantes, los factores más relevantes y relacionados con altos niveles de logro son: la inteligencia, la motivación y los hábitos de estudio. (Borges, Hernández \& González, 2012)

Se analizaron las asociaciones entre burnout académico y engagement académico, esto permitió considerarlos como polos extremos de un mismo fenómeno, conformado por las dimensiones: vigor, dedicación, ineficacia académica y cinismo. Se identificaron variables sociodemográficas y académicas, donde el burnout académico resultó un buen predictor de mal desempeño académico y el engagement académico un buen pronosticador de buen desempeño académico. (Caballero, Hederich y García, 2015).

Otra investigación evidenció que los alumnos con mayores niveles de engagement eran mujeres, las que ingresaron con altas notas en números, se comprobó que desarrollaban más actividades al interior de la universidad, al mismo tiempo tenían mejores calificaciones. (Parada \& Pérez, 2014).

Contextualizando, el engagement académico, ha demostrado ser un factor fundamental para impulsar los aprendizajes, rendimiento, interés, disfrute y bienestar psicológico en alumnos. Sin embargo, aún quedan en evidencia ciertas fisuras conceptuales que deben profundizarse y esclarecerse. Finalmente Medrano \& Adrián, (2015), concluyen que la definición de engagement basadas en factores de activación y dirección resulta en un constructo modélico bien fundamentado.

Los estudiantes que muestran menores tasas de rendimiento académico, menor apoyo de los padres y bajos recursos de familia, enfrentan su progreso profesional en condiciones altamente desfavorables. (Park, Rojewski \& Lee, 2018).

Existe considerable evidencia de estudios realizados respecto a los factores que determinan en gran medida el rendimiento académico en estudiantes de diversos niveles académicos, los mismos que señalan que una de las variables más importantes determinantes en el desenvolvimiento académico es la satisfacción emocional-psicológica y presentan relación con la calificación final obtenida por los estudiantes en el tema. (Guardia et al., 2006).

En el mismo sentido, y a efectos de lograr un desempeño académico óptimo, las investigaciones señalan que los educandos deben involucrarse con su aprendizaje, por ello, el engagement académico demuestra ser importantísimo para "promover el aprendizaje, rendimiento, interés, disfrute y bienestar psicológico de los estudiantes”. (Medrano \& Adrián, 2015).

Otros estudios, señalan que el apego favorable a requerimientos académicos se visibiliza en tanto mayor sea el engagement de los estudiantes en su continuo educativo. En tal sentido, las convicciones de autoeficacia académica percibida y el involucramiento emocional con labores académicas son predictores de lealtad y engagement académico. (Cavazos \& Encinas, 2016).

Se afirma también, que la relación del engagement con el desempeño en ámbitos laborales y educacionales, así como su relevancia como elemento motivacional en la preparación de alumnos independientes (Parada \& Pérez, 2014).

Las altas expectativas educacionales de la familia son el factor diferenciador más importante en el rendimiento escolar (Bravo et al., 2017).

En esta sección, se pasa a detallar el análisis realizado en el presente estudio, al tiempo que consideramos que, en el contexto de la educación universitaria, se deben profundizar aquellos estudios que permitan identificar fehacientemente los factores determinantes de motivación y energía positiva para el estudio, debido a que en la actualidad los estudiantes aprenden de manera lúdica y poco convencional desafiando en muchos casos la lógica formal y reglamentaria del sistema educativo.

$\mathrm{Al}$ mismo tiempo, algunos autores refieren que la definición del término engagement todavía requiere mucho debate y entendimiento, por ende la descripción detallada de sus características es importante, a fin de nutrir el estado del arte de dicha variable (Compagnucci, Cardós, Denegri \& Barboza, 2002). 
Las investigaciones referidas a la asociación entre engagement y rendimiento académico deben profundizarse aún más.

Se debe precisar que la variable engagement, en nuestra realidad, es estudiada más dentro del contexto empresarial; por ende, dentro de los círculos académicos su nivel de incidencia es menor, por no haber multiplicidad de estudios en esos entornos.

Se estableció la relación existente entre engagement y rendimiento académico. Así mismo, el objetivo fue describir la relación entre engagement y rendimiento académico.

Se comprobó la asociación relacional entre vigor y rendimiento académico; el objetivo específico 1, fue describir la relación entre vigor y rendimiento académico.

Se determinó la asociación relacional entre dedicación rendimiento académico; el objetivo 2, fue describir la relación entre dedicación y rendimiento académico.

Se estableció la asociación relacional entre absorción y rendimiento académico; el objetivo 3 , fue describir la relación entre absorción y rendimiento académico.

La hipótesis, buscó establecer la relación significativa entre engagement y rendimiento académico, a partir de lo cual se podrían delinear programas apropiados y modelos de gestión que permitan optimizar las estrategias de enseñanza y gestión educativa rediciendo la deserción estudiantil.

\section{Materiales y métodos}

SLa investigación se realizó en el ámbito de la Facultad de Ciencias Aplicadas de la Universidad Nacional del Centro del Perú, con estudiantes de las carreras profesionales de administración de negocios, administración hotelera y turismo e ingeniería agroindustrial, en Pomachaca Tarma Junín Perú.

El estudio fue de nivel correlacional, el cual se operativizó a través de la escala validada internacionalmente UWES de engagement, dirigida a la totalidad de estudiantes presentes de la Facultad de Ciencias Aplicadas de la Universidad Nacional del Centro del Perú, se aplicó en el año 2018.

La población-muestra, se compuso de 298 alumnos, se encuestaron en un solo acto a todos ellos a través del cuestionario validado a nivel internacional de engagement académico UWES. La escala estuvo conformada por los siguientes criterios: Siempre $=5$, Casi Siempre $=4$, A Veces $=3$, Casi Nunca $=2$, Nunca $=1$; tal nomenclatura, permitió determinar los porcentajes de variación entre un engagement elevado 5 y un engagement muy bajo 1 .

De acuerdo a la ficha de matrículas de la Facultad de Ciencias Aplicadas, se tiene 297 estudiantes.

Los criterios de inclusión comprendieron a todos los alumnos de las tres carreras profesionales de la Facultad de Cien- cias Aplicadas. El principal criterio de exclusión fue que no se consideraron a los estudiantes que estuvieron de licencia o permiso el día en que se recolectó la data e información.

Los cuestionarios fueron aplicados de manera personal en formato físico (papel), durante los horarios de clases acordados y, fueron aplicados directamente por los responsables del proyecto y autores del presente artículo. El instrumento era anónimo pudiendo los alumnos declinar en caso no estén conformes.

El cuestionario UWES, operacionaliza el concepto de engagement académico, desagregándolo en tres dimensiones: Vigor, Dedicación y Absorción, es un instrumento validado a nivel internacional. (Medrano \& Adrián, 2015).

Con el objeto de comprobar la asociación de variables, se empleó el coeficiente de correlación de Spearman para muestras no paramétricas cualitativas categóricas, el nivel de significancia fue de $5 \%$, y el de confianza fue de $95 \%$, los datos fueron procesados en el paquete estadístico SPSS 22.

Los estudiantes fueron convocados e informados por los investigadores, obteniéndose los permisos pertinentes. El proceso tuvo en consideración consideraciones éticas nacionales e internacionales. Fue aprobado por el comité de ética de la universidad.

El propósito principal, fue determinar la relación entre engagement y rendimiento académico en estudiantes de la Facultad en estudio.

Dada la naturaleza transeccional del presente estudio, se estableció la correlación como objetivo principal de la investigación

\section{Resultados}

El más resaltante y evidente hallazgo encontrado, es que no existe correlación significativa entre engagement y rendimiento académico, a partir del cual se contrasta con la evidencia empírica, existiendo un nivel apropiado de motivación y responsabilidad en los estudiantes de la Facultad respecto a sus estudios.

Al comprobar la hipótesis, se busca demostrar la existencia de correlación significativa entre engagement y rendimiento académico en estudiantes de la Facultad estudiada; asimismo, se obtuvo un p-valor de 0.33 , por tanto, inferimos que no existe correlación significativa entre engagement y rendimiento académico, también el coeficiente de correlación 0.123 , indica un nivel bajo de asociación entre variables.

En la Tabla 1, se observa que el mejor rendimiento académico lo tuvieron los estudiantes del IX semestre, con 13.19 en promedio; seguido delVII semestre, con 12.36; V semestre, con 11.90 y; el III semestre, con 10.40. Así mismo, los estudiantes de la Escuela Profesional de Ingeniería Agroindustrial tuvieron un promedio de 12.51 . 
Tabla 1

Estadísticos descriptivos del rendimiento académico - Ingeniería Agroindustrial

\begin{tabular}{cccccc}
\cline { 2 - 6 } & N & Mínimo & Máximo & Media & $\begin{array}{c}\text { Desv. } \\
\text { estándar }\end{array}$ \\
\hline III semestre & 4 & 4.00 & 14.00 & 10.40 & 4.40 \\
V semestre & 24 & 4.81 & 14.88 & 11.90 & 1.73 \\
VII semestre & 16 & 2.30 & 14.27 & 12.36 & 2.79 \\
IX semestre & 38 & 11.00 & 15.00 & 13.19 & 0.94 \\
AIT & $\mathbf{8 2}$ & $\mathbf{2 . 3 0}$ & $\mathbf{1 5 . 0 0}$ & $\mathbf{1 2 . 5 1}$ & $\mathbf{1 . 9 9}$ \\
\hline
\end{tabular}

En la Tabla 2, se observa que el mejor rendimiento académico lo tuvieron los estudiantes del V semestre con 14.46 en promedio; seguido del IX semestre, con 14.06; VII semestre, con 13.39 y; el III semestre; con 13.32. Así mismo, todos los estudiantes de la Escuela Profesional de Administración Hotelera y Turismo tienen un promedio de 13.87.

Tabla 2

Estadísticos descriptivos del rendimiento académico - Administración Hotelera y Turismo

\begin{tabular}{cccccc}
\cline { 2 - 6 } & N & Mínimo & Máximo & Media & $\begin{array}{c}\text { Desv. } \\
\text { estándar }\end{array}$ \\
\hline III semestre & 5 & 12.39 & 14.36 & 13.32 & 0.71 \\
V semestre & 21 & 12.41 & 15.55 & 14.46 & 0.86 \\
VII semestre & 29 & 4.00 & 16.04 & 13.39 & 2.20 \\
IX semestre & 23 & 6.55 & 17.41 & 14.06 & 2.48 \\
HTT & $\mathbf{7 8}$ & $\mathbf{4 . 0 0}$ & $\mathbf{1 7 . 4 1}$ & $\mathbf{1 3 . 8 7}$ & $\mathbf{1 . 9 9}$ \\
\hline
\end{tabular}

En la Tabla 3, se observa que el mejor rendimiento académico lo tuvieron los estudiantes del IX semestre, con 14.67 en promedio; seguido del V semestre, con 13.68; III semestre, con 13.58 y; el III semestre, con 13.32. Además, los estudiantes de la Escuela Profesional de Administración de Negocios tienen un promedio de 13.79.

\section{Tabla 3}

Estadísticos descriptivos del rendimiento académico - Administración de Negocios

\begin{tabular}{cccccc}
\cline { 2 - 6 } & N & Mín. & Máx. & Media & $\begin{array}{c}\text { Desv. } \\
\text { estándar }\end{array}$ \\
\hline III semestre & 23 & 9.81 & 15.23 & 13.58 & 1.27 \\
V semestre & 49 & 11.67 & 16.05 & 13.68 & 1.06 \\
VII semestre & 36 & 4.86 & 17.00 & 13.32 & 2.70 \\
IX semestre & 30 & 12.82 & 16.18 & 14.67 & 0.73 \\
ANT & $\mathbf{1 3 8}$ & $\mathbf{4 . 8 6}$ & $\mathbf{1 7 . 0 0}$ & $\mathbf{1 3 . 7 9}$ & $\mathbf{1 . 7 0}$ \\
\hline
\end{tabular}

En la Tabla 4, se observa que de los 298 estudiantes de la FACAP UNCP de las 3 carreras profesionales en estudio, se observa una nota mínima de 2.30 y una máxima de 17.41 , con una media de 13.46 y una desviación estándar de 1,94.
Tabla 4

Estadísticos descriptivos, rendimiento académico de estudiantes de la Facultad de Ciencias Aplicadas

\begin{tabular}{llllll}
\cline { 2 - 5 } & N & Mínimo & Máximo & Media & $\begin{array}{c}\text { Desv. } \\
\text { estándar }\end{array}$ \\
\hline $\begin{array}{l}\text { Prom. Notas. } \\
\text { FACAP }\end{array}$ & 298 & 2.30 & 17.41 & 13.4584 & 1.94231 \\
\hline N válido por lista $=298$ & & & & \\
\hline
\end{tabular}

Los datos permiten afirmar que estamos ante una población-muestra no paramétrica propia de las variables categóricas. La prueba de Kolmogorov - Smirnov se muestra en la Tabla 5.

Tabla 5

Prueba de Kolmogorov - Smirnov de engagement y rendimiento académico

\begin{tabular}{cccccccc}
\cline { 2 - 7 } & \multicolumn{3}{c}{$\begin{array}{c}\text { Kolmogor- } \\
\text { ov-Smirnov }^{\text {a }}\end{array}$} & \multicolumn{3}{c}{ Shapiro-Wilk } \\
\cline { 2 - 7 } & Estadístico & gl & Sig. & Estadístico & gl & Sig. \\
\hline $\begin{array}{c}\text { Engagement } \\
\text { FACAP }\end{array}$ & .289 & 298 & $\mathbf{0 . 0 0 0}$ & .808 & 298 & $\mathbf{0 . 0 0 0}$ \\
$\begin{array}{c}\text { Prom. Notas } \\
\text { FACAP. Tablas }\end{array}$ & .368 & 298 & $\mathbf{0 . 0 0 0}$ & .705 & 298 & $\mathbf{0 . 0 0 0}$ \\
\hline
\end{tabular}

En la Tabla 6, con un p-valor del $33 \%$, se concluye que no existe correlación significativa entre el engagement y rendimiento académico en estudiantes de la Facultad de Ciencias Aplicadas de la UNCP.

\section{Tabla 6}

Correlación de Spearman, engagement y rendimiento académico en estudiantes de la Facultad

\begin{tabular}{ccc}
\hline Variables & p-valor & $\begin{array}{c}\text { Coeficiente } \\
\text { de correlación }\end{array}$ \\
\hline $\begin{array}{c}\text { Engagement y } \\
\text { rendimiento académico }\end{array}$ & 0.33 & 0.123 \\
\hline
\end{tabular}

*. La correlación es significativa en el nivel 0,05 (bilateral).

Con un p-valor del $29 \%$ (Tabla 7), se concluye que no existe correlación significativa entre vigor y rendimiento académico en estudiantes de la Facultad de Ciencias Aplicadas de la UNCP.

Tabla 7

Correlación de Spearman, vigor y rendimiento académico en estudiantes de la Facultad

\begin{tabular}{lcc}
\hline Dimensión de variable & p-valor & $\begin{array}{c}\text { Coeficiente } \\
\text { de correlación }\end{array}$ \\
\hline $\begin{array}{c}\text { Dim. Vigor de engagement } \\
\text { y rendimiento académico }\end{array}$ & 0.29 & .127 \\
\hline
\end{tabular}

Con un p-valor del $11.5 \%$ (Tabla 8), se concluye que no existe correlación significativa entre dedicación y rendimiento académico en estudiantes de la Facultad de Ciencias Aplicadas de la UNCP. 


\section{Tabla 8}

Correlación de Spearman, dedicación y rendimiento académico en estudiantes de la FACAP

\begin{tabular}{ccc}
\hline Dimensión de variable & p-valor & $\begin{array}{c}\text { Coeficiente } \\
\text { de correlación }\end{array}$ \\
\hline $\begin{array}{c}\text { Dim. Dedicación de en- } \\
\text { gagement y rendimiento } \\
\text { académico }\end{array}$ & 0.115 & .091 \\
\hline
\end{tabular}

Con un p-valor del $38 \%$, se concluye que no existe correlación significativa entre el engagement y rendimiento académico en estudiantes de la Facultad de Ciencias Aplicadas de la UNCP (Tabla 9).

Tabla 9

Correlación de Spearman, absorción y rendimiento académico en estudiantes de la FACAP

\begin{tabular}{ccc}
\hline Dimensión de variable & p-valor & $\begin{array}{c}\text { Coeficiente } \\
\text { de correlación }\end{array}$ \\
\hline $\begin{array}{c}\text { Dim. Absorcion de enga- } \\
\text { gement y rendimiento } \\
\text { académico }\end{array}$ & 0.38 & .120 \\
\hline
\end{tabular}

\section{Discusión}

Respecto al propósito principal del estudio, se afirma que el engagement no tiene relación significativa con el rendimiento académico, lo cual evidencia que los estudiantes participantes al momento de realizar el estudio, presentan niveles moderados de resiliencia (sobreposición frente a la adversidad), pese que existen condiciones adversas o limitaciones; sin embargo, muestran mucho interés por aprender, estudiar e investigar. Estos hallazgos reafirman lo expresado por Fenollar, Román y Cuestas (2008), quienes en su estudio concluyen y señalan que no existe relación significativa entre resultados esperados y calificaciones promedio; al mismo tiempo, los resultados obtenidos, refutan lo referido por Harackiewicz, Barron, Tauer, Carter y Elliot (2000), quienes señalan que: la combinación de ambas variables, pueden tener consecuencias beneficiosas dentro del contexto educativo, al mismo tiempo Horstmanshof \& Zimitat (2007), citando a Martin, (2008) y Salanova, Bresó, \& Schaufeli (2005), concluyen que el engagement académico ha demostrado ser un elemento neurálgico en la promoción del aprendizaje, aspectos psicológicos positivos a nivel psicosomático de los estudiantes.

En lo concerniente a la dimensión vigor de engagement y rendimiento académico, no se correlacionan significativamente al haber obtenido un p-valor de 0.29 y un coeficiente de correlación de Spearman de 0.127, contradiciendo lo establecido por Parra (2011), quien refiere que la satisfacción se conceptualizó como una dimensión del engagement, favoreciendo al rendimiento del estudiante y un mejor afrontamiento de situaciones estresantes.
En relación a la dimensión dedicación de engagement y rendimiento académico, tampoco se encuentra correlación significativa con un p-valor de 0.115 y un coeficiente de correlación de Spearman de 0.91, refutando lo expresado por Gonzáles, Caso, Díaz \& López (2012), citando a De la Orden, Oliveros, Mafokozi y González (2001); Edel (2003) y; Martínez-Otero (2009), quienes precisan que el rendimiento académico tiene que ver principalmente con reto, logro y resultados académicos.

Finalmente, y con respecto a la dimensión absorción de engagement y rendimiento académico, se obtuvo un p-valor de 0.38 y un coeficiente de correlación de Spearman 0.120, no existiendo correlación significativa, discrepando con Cominetti y Ruiz (1997), citado por Edel (2003), quienes refieren que las expectativas del entorno directo del estudiante permiten sacar a relucir los paradigmas positivos y negativos que acompañan al estudiante y que serán determinantes en su rendimiento y logros académicos.

De lo anterior, y al no haber relación significativa entre engagement y rendimiento académico, podría preverse que en el futuro y de manera súbita e inesperada los estudiantes pierdan el interés en sus estudios y en consecuencia bajen sus calificaciones cíclicamente o abandonen sus estudios.

$\mathrm{Al}$ respecto, las principales limitaciones que se presentaron durante la ejecución del estudio fue que la población-muestra fue aplicada a estudiantes de una sola Facultad, por lo que los resultados no podrían ampliarse a otros contextos, pues no sería evidencia representativa, por lo que se sugiere realizar más estudios similares con posterioridad.

\section{Conclusiones}

- La investigación ha demostrado, a través de los resultados de las encuestas y el análisis estadístico, que existe relación débil entre el engagement estudiantil y el rendimiento académico en la FACAP - UNCP.

- Con un coeficiente de relación de 0,123; se afirma que engagement académico se relaciona de manera débil con el rendimiento académico; en concordancia con los resultados, se puede concluir, en términos generales, que el engagement de los estudiantes en el año lectivo 2018 - I ha sido débil en la Facultad de Ciencias Aplicadas para estar relacionado con el rendimiento de los alumnos de las tres carreras profesionales.

- Respecto a la dimensión vigor, como altos niveles de energía y resiliencia, voluntad de dedicar esfuerzos, no fatigarse con facilidad y persistencia ante las dificultades; y, según los resultados, esto tiene que ser fortalecida con clases motivacionales y de crecimiento profesional dentro de los cursos del plan de estudios y fuera de ellos para mejorar el panorama de esta dimensión estudiada. 
- Con relación a la dimensión dedicación como: sentido o significado del trabajo, sentirse entusiasmado, orgullo por su labor y sentirse inspirado por el trabajo y de acuerdo a los resultados, se tiene que fortalecer este tema con el trabajo que se realiza en tutoría para mejorar los niveles de dedicación en sus labores académicas.

- En cuanto a la dimensión absorción, como felizmente inmerso en su trabajo, presenta dificultad para dejarlo, el tiempo pasa rápidamente y uno se olvida de lo que pasa a su alrededor y, según resultados, se debe mejorar estos niveles con charlas personales a los estudiantes.

- Se concluye, que la investigación tiene que pasar a un siguiente nivel para poder explicar el origen de la relación débil de las variables estudiadas y sus posibles causas.

\section{Referencias bibliográficas}

Berbosa Honrado, R. (2017). Estrés académico, engagement y resiliencia en estudiantes de enfermería. Valladolid: Universidad de Valladolid.

Blanco Pineda, J. (15 de febrero de 2018). s/n. Obtenido de s/n: http://ri.ufg.edu.sv/jspui/bitstream/1159 2/6360/3/371.262-B634f-CAPITULO\%20II.pdf

Blanco, Córdova \& Guerrero. (2005). Factores psicosociales que afectan el rendimiento académico de los estudiantes de bachillerato de los Institutos José Damián Villacorta, Walter Soundy y los Colegios Santa Inés e Inmaculada Concepción turno diurno del Municipio de Santa Tecla. San Salvador: Universidad Francisco Gavidia.

Borges del Rosal, Á., Hernández-Jorge, C. M. \& González Sierra, M. A. (2012). Achievement predictors in a secondary students' sample. Quality \& Quantity, 46(6), 1687-1697. https://doi.org/10.1007/ s11135-011-9547-5

Bravo Sanzana, M., Salvo, S., Mieres, M., Mansilla, J., Hederich, C., Bravo Sanzana, M., ... Hederich, C. (2017). Perfiles de desempeño académico: la importancia de las expectativas familiares. Perfiles Latinoamericanos, 25(50), 361-386. https://doi. org/10.18504/pl2550-016-2017

Caballero D., Carmen Cecilia; Hederich G., Christian; García P., A. (2015). Relación entre burnout y engagement académicos con variables sociodemográficas y académicas. Psicología desde el Caribe, 32(2), 254-267. Retrieved from http://www.redalyc. org / articulo.oa?id=21341030005

Caballero, Abello \& Palacio. (2006). Burnout, engage- ment y rendimiento académico entre estudiantes universitarios que trabajan y aquellos que no trabajan. Psicogente, 11-27.

Caballero, Hederich \& Garcia. (2015). Relación entre burnout y engagement académicos con variables sociodemograficas y academicas. Psicología desde El Caribe, Vol. 32, n. ${ }^{\circ}$ 2, 254-267.

Caruso Holgado, M. J. (2011). Estudio del estrés, engagement y rendimiento académico en estudiantes universitarios de Ciencias de la Salud. Malaga: Universidad de Málaga, Servicio de Publicaciones.

Cavazos Arroyo, J. \& Encinas Orozco, F. C. (2016). Influencia del engagement académico en la lealtad de estudiantes de posgrado: un abordaje a través de un modelo de ecuaciones estructurales. Estudios Gerenciales, 32(140), 228-238. https://doi. org/10.1016/j.estger.2016.07.001

Compagnucci, E., Cardós, P., Denegri, A. \& Barboza, C. (2002). El adolescente frente al conocimiento. Orientación y Sociedad, 3, 93-104. Retrieved from http: / / www.scielo.org.ar/scielo.php?script $=$ sci_arttext\&pid =S1851-88932002000100006\&lng =es\&nr$\mathrm{m}=\mathrm{iso} \& \mathrm{t} \operatorname{lng}=\mathrm{es}$

Córdoba Reyes, N. J. (2015). Niveles de engagement en los colaboradores de una industria de detergentes ubicadas en Escuintla. Escuintla: Universidad Rafael Landívar.

Edel Navarro, R. (2003). El rendimiento académico: concepto, investigación y desarrollo. REICE. Revista Iberoamericana sobre Calidad, Eficacia y Cambio en Educación, 0.

Erazo, O. (2012). El rendimiento académico, un fenómeno de multiples relaciones y complejidades. Revista Vanguardia Psicológica Clínica Teórica y Práctica; Volumen 2, Número 2, 144-173.

Fenollar, Cuestas \& Román. (2008). Antecedentes del rendimiento académico: Aplicacion a la docencia en marketing. Revista Española de Investigación de Marketing ESIC; Vol. 12, n. 2 (7-24), 7-25.

Garbanzo Vargas, G. M. (2007). Factores asociados al rendimiento académico en estudiantes universitarios, una reflexión desde la educación. Revista Educación, vol. 31, núm. 1, 43-63.

García Rodriguez, J. J. (2013). Estrés, engagement y rendimiento académico de los estudiantes de enfermería en las prácticas clínicas. Málaga: Universidad de Málaga.

Garzon, Rojas, Riesgo, Pinzon \& Salamanca. (2010). Factores que pueden influir en el rendimiento aca- 
démico de estudiantes de bioquímica que ingresan en el Programa de Medicina de la Universidad del Rosario Colombia. EDUC MED 2010; 13 (2): 85-96 Viguera Editores SL, 85-96.

Gonzáles, Caso, Diaz \& López. (2012). Rendimiento académico y factores asociados. Aportaciones de algunas instituciones a gran escala. Bordón 64 (2), 2012, 51-68.

Gonzáles, Hernández, Soberanes \& Sanchez. (s.f.). Estudio del burnout y engagement académico en estudiantes universitarios. Primer Congreso Internacional de Educacion "Construyendo Ineditos Viables".

Guàrdia, J., Freixa, M., Peró, M., Turbany, J., Cosculluela, A., Barrios, M., \& Rifà, X. (2006). Factors related to the academic performance of students in the statistics course in psychology. Quality \& Quantity, 40(4), 661-674. https://doi.org/10.1007/ s11135-005-2072-7

Holgado, C. \& Jesús. (2011). Estudio del estres, engagement y rendimiento en estudiantes universitarios de Ciencias de la Salud. Málaga: Servicio de Publicaciones de la Universidad de Málaga.

Lavaggi. (2017). Cansancio emocional y engagement en estudiantes de una universidad privada de Lima. Lima: Universidad Peruana de Ciencias Aplicadas.

Marroquin. (15 de febrero de 2018). s/n. Obtenido de s/n: http://www.une.edu.pe/Sesion04-Metodologia_de_la_investigacion.pdf

Martínez \& Salanova. (2003). Niveles de Burnout y engagement en estudiantes universitarios. Relación con el desempeño y desarrollo profesional. Revista de Educación. núm. 330, 361-384.

Medrano, P. \& Adrián, L. (2015). Medición del engagement académico en estudiantes universitarios, 2, 114-124. Retrieved from http: / / www.redalyc.org/articulo. oa?id=459645432012

Medrano, Moreti \& Ortiz. (2015). Medición del engagement académico en estudiantes universitarios Revista Iberoamericana de Diagnóstico y Evaluación, 114-123.

Ocaña Fernández, Y. (2011). Variables académicas que influyen en el rendimiento académico de los estudiantes universitarios. Investigación Educativa. Vol. 15 N. ${ }^{\circ} 27,165-179$.

Orezolli Hoyle, C. E. (2017). Engagement académico en estudiantes de clases espejo en una universidad privada del Perú, durante el año 2016. Trujillo: Universidad Privada del Norte.
Parada \& Pérez. (2013). Relación del engagement académico con características académicas y socioafectivas en estudiantes de Odontología. Educ Med Super, 199-215.

Parada Contreras, M. \& Pérez Villalobos, C. E. (2014). Relación del engagement académico con características académicas y socioafectivas en estudiantes de odontología. Educación Médica Superior, 28(2), 199-215. Retrieved from http:// scielo.sld.cu/scielo.php?script $=$ sci_arttext\&pi$\mathrm{d}=$ S0864-21412014000200003\&lng=es\&nr$\mathrm{m}=\mathrm{iso} \& \mathrm{t} \operatorname{lng}=\mathrm{es}$

Park, J.-H., Rojewski, J. W., \& Lee, I. H. (2018). Determinants of adolescents' career development competencies in junior secondary schools of South Korea. International Journal for Educational andVocational Guidance, 18(1), 1-25. https://doi.org/10.1007/ s10775-017-9342-5

Rojas, E. (2000). El Hombre Light: Una vida sin valores. Buenos Aires: Planeta.

Tellez \& Torres. (2013). Desempeño académico, burnout y engagement en los estudiantes de la Facultad de Ciencias Médicas de la UNAN. Leon: Universidad Nacional Autónoma de Nicaragua, León.

Vásquez Roca, A. (2008). Zygmunt Bauman: Modernidad liquida y fragilidad humana. Nomadas: revista de criticade ciencias sociales y juridicas, 1.

Vizoso \& Arias. (2016). Engagement, burnout y rendimiento académico en estudiantes universitarios y su relación con la prioridad en la eleccion de la carrera. Revista de Psicología y Educación, 11(1), 45-60. 